A

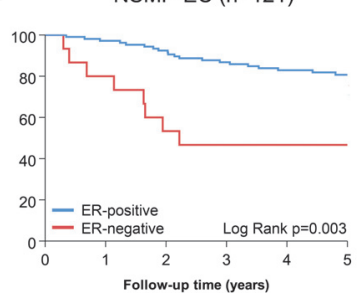

B ER-positive-NSMP EC ( $n=106)$

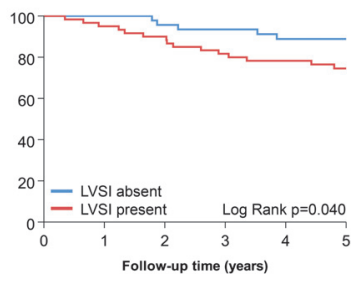

Abstract 397 Figure 2 Time to recurrence within NSMP-EC

and p53-abnormal immunohistochemistry (IHC). Protein expression of L1CAM, ER and PR (ongoing) were analysed by IHC using a $10 \%$ threshold for positivity. Tumour DNA was analysed for pathogenic somatic mutations using a next generation sequencing (NGS) cancer hotspot panel. Time to recurrence was analysed using the Kaplan-Meier method, logrank tests and Cox's proportional hazard models.

Result(s)* In total, 126 NSMP-EC were identified in PORTEC-3, the majority were hormone receptor positive (ER $\mathrm{n}=106 / 121,87.6 \%$, PR will be presented at ESGO2021). L1CAM overexpression was observed in $11.2 \% \quad(n=14 / 125)$ and mutations in CTNNB1-exon-3 were identified in $34.3 \%$ $(n=36 / 105)$. Clustering showed that ER-positive NSMP-EC were predominantly endometrioid EC ( $\mathrm{n}=99$, 93.4\%), low grade $(\mathrm{n}=88,83.0 \%)$ and L1CAM-negative $(\mathrm{n}=102,97.1 \%)$ (figure 1). PIK3CA and KRAS mutations were present in $27.3 \% \quad(n=24)$ and $19.3 \%(n=17)$, respectively. ER-negative NSMP-EC were frequently non-endometrioid ( $\mathrm{n}=11,73.3 \%)$, L1CAM-positive $(\mathrm{n}=11,73.3 \%)$ and rarely harboured PTEN and CTNNB1 mutations $(\mathrm{n}=1,7.1 \%$ and $0 \%$, respectively). ER-positivity was associated with lower risk of recurrence (HR 0.32 [95\%CI 0.14-0.70]; figure 2A), while L1CAM-overexpression and CTNNB1-exon-3 mutations were not (HR 2.25 [95\%CI 0.93-5.43] and HR 1.20 [95\%CI 0.57-2.54], respectively). Multivariable analysis confirmed independent favourable prognostic impact of ER-positivity and LVSI. Figure 2B shows impact of LVSI on time to recurrence among patients with ER-positive NSMP-EC.

Conclusion* The vast majority of NSMP-HREC are ER-positive $(87.6 \%)$ and are likely sensitive to hormonal therapy. Other treatment targets might be found in this subgroup too as $27.3 \%$ had PIK3CA and $19.3 \%$ had KRAS mutations. NSMP-EC with loss of ER-expression were often of non-

endometrioid histology and had a high risk of recurrence. Future studies should investigate whether this subgroup would benefit from other systemic therapies.

\section{PROGNOSTIC RELEVANCE OF THE MOLECULAR ENDOMETRIAL CANCER CLASSIFICATION AMONG PATIENTS STAGED BY LYMPHADENECTOMY AND/OR WITHOUT ADJUVANT TREATMENT}

${ }^{1} \mathrm{~A}$ Leon-Castillo*, ${ }^{2} \mathrm{~N}$ Horeweg, ${ }^{1} \mathrm{E}$ Peters, ${ }^{1} \mathrm{~T}$ Rutten, ${ }^{1} \mathrm{~N}$ Ter Haar, ${ }^{1} \mathrm{~V}$ Smit, ${ }^{3 ; 4} \mathrm{M}$ Boennelycke, ${ }^{3 ; 4} \mathrm{E}$ Høgdall, ${ }^{5} \mathrm{C}$ Høgdall, ${ }^{2} \mathrm{R}$ Nout, ${ }^{2} \mathrm{CL}$ Creutzberg, ${ }^{5} \mathrm{G}$ Ortoft, ${ }^{1 ; 6} \mathrm{~T}$ Bosse. 'Leiden University Medical Center (LUMC), Pathology, Leiden, Netherlands; '2Leiden University Medical Center (LUMC), Radiation oncology, Leiden, Netherlands; ${ }^{3}$ Herlev and Gentofte Hospital, Pathology, Herlev, Denmark; ${ }^{4}$ Copenhagen University Hospital, Rigshospitalet, Pathology, København, Denmark; ${ }^{5}$ Copenhagen University Hospital, Rigshospitalet, Gynecology, København, Denmark; ${ }^{6}$ Leiden University Medical Center, Department of Pathology, Leiden, Netherlands

\subsection{6/ijgc-2021-ESGO.138}

Introduction/Background* The molecular endometrial cancer (EC) classification has proven prognostic impact. However, patients included in previous studies were not always staged by lymphadenectomy (LND) and often received adjuvant treatment (AT). This may have moderated the prognostic effect of the molecular classification. We evaluated the prognostic significance of the molecular classification in high-grade EC patients staged by LND and those without AT.

Methodology Targeted DNA-sequencing for pathogenic POLEexonuclease domain mutations and immunohistochemistry for mismatch repair (MMR) proteins and p53 expression were performed on 412 high-grade EC from the Danish Gynecological Cancer Database 2005-2012 to classify them as POLEultramutated (POLEmut), MMR-deficient (MMRd), p53mutant (p53abn), or no specific molecular profile (NSMP). Patients with stage IV or residual disease after surgery were excluded. Analyses were performed on patients staged by LND and on patients without AT. Time to recurrence analyses were performed using the Kaplan-Meier method, log-rank test and Cox proportional hazard's models. Pre-specified multivariate regression analyses were performed including age, ASA class, stage, lymphovascular space invasion and in the LND subgroup a propensity score to correct for confounding by indication.
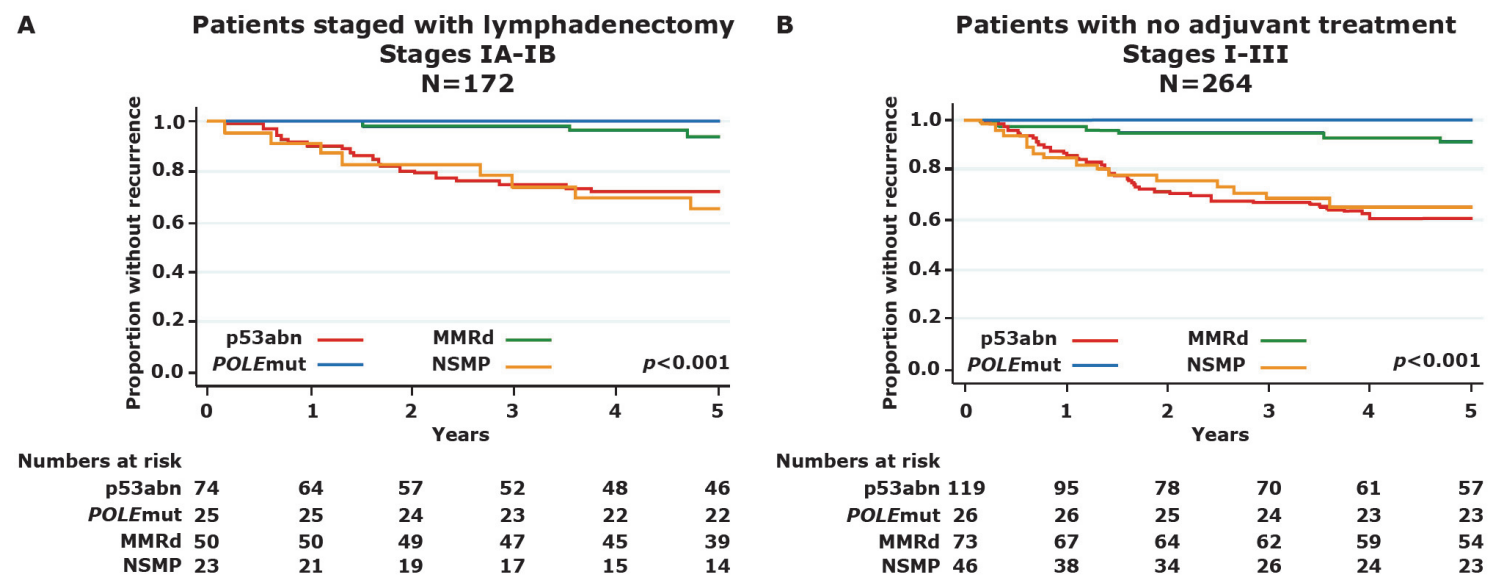

Abstract 406 Figure 1 Time to recurrence of patients staged by lymphadenectomy as stage IA-IB, and of patients with no adjuvant tratment 
Abstract 406 Table 1 Clinicopathological features of patients with high grade endometrial cancer staged by lymphadenectomy

\begin{tabular}{|c|c|c|c|c|c|c|c|}
\hline & & $\begin{array}{r}\text { Total } \\
\mathrm{n}=251 \\
(\%) \\
\end{array}$ & $\begin{array}{r}\text { p53abn } \\
n=108 \\
(43.0 \%) \\
\end{array}$ & $\begin{array}{r}\text { POLEmut } \\
n=27 \\
(10.8 \%) \\
\end{array}$ & $\begin{array}{r}\text { MMRd } \\
n=78 \\
(31.1 \%) \\
\end{array}$ & $\begin{array}{r}\text { NSMP } \\
n=38 \\
(15.1 \%) \\
\end{array}$ & $P$ \\
\hline \multirow[t]{3}{*}{ Age, years } & & & & & & & 0.05 \\
\hline & $0-69$ & $152(60.6)$ & $56(51.9)$ & $23(85.2)$ & $50(64.1)$ & $23(60.5)$ & \\
\hline & $>70$ & $99(39.4)$ & $52(48.1)$ & $4(14.8)$ & $28(35.9)$ & $15(39.5)$ & \\
\hline \multirow[t]{5}{*}{ Histotype } & & & & & & & $<0.001$ \\
\hline & Endometrioid grade 3 & $107(42.6)$ & $25(23.1)$ & $16(59.3)$ & $48(61.5)$ & $18(47.4)$ & \\
\hline & Serous & $87(34.7)$ & $61(56.5)$ & $9(33.3)$ & $9(11.5)$ & $8(21.1)$ & \\
\hline & Clear cell & $51(21.3)$ & $18(16.7)$ & $2(7.4)$ & $19(24.4)$ & $12(31.6)$ & \\
\hline & Undifferentiated & $6(2.4)$ & $4(3.7)$ & $0(0)$ & $2(2.6)$ & $0(0)$ & \\
\hline \multirow[t]{7}{*}{ Stage } & & & & & & & 0.21 \\
\hline & IA & $115(45.8)$ & $53(49.1)$ & $15(55.6)$ & $34(43.6)$ & $13(34.2)$ & \\
\hline & IB & $57(22.7)$ & $21(19.4)$ & $10(37.0)$ & $16(20.5)$ & $10(26.3)$ & \\
\hline & II & $19(7.6)$ & $6(5.6)$ & $1(3.7)$ & $7(9.0)$ & $5(13.2)$ & \\
\hline & IIIA & $1(0.4)$ & $0(0)$ & $0(0)$ & $1(1.3)$ & $0(0)$ & \\
\hline & IIIB & $8(3.2)$ & $1(0.9)$ & $0(0)$ & $5(6.4)$ & $2(5.3)$ & \\
\hline & IIIC & $51(20.3)$ & $27(25.0)$ & $1(3.7)$ & $15(19.2)$ & $8(21.1)$ & \\
\hline \multicolumn{2}{|c|}{ Lymphovascular space invasion } & & & & & & 0.64 \\
\hline & Absent/focal & $210(83.7)$ & $89(82.4)$ & $25(92.6)$ & $63(80.7)$ & $33(86.8)$ & \\
\hline & Substantial & $35(13.9)$ & $15(13.9)$ & $2(7.4)$ & $14(17.9)$ & $4(10.5)$ & \\
\hline & Missing & $6(2.4)$ & $4(3.7)$ & $0(0)$ & $1(1.3)$ & $1(2.3)$ & \\
\hline \multicolumn{2}{|c|}{ Adjuvant treatment received } & & & & & & 0.61 \\
\hline & None & $178(70.9)$ & $77(71.3)$ & $22(81.5)$ & $53(67.9)$ & $26(68.4)$ & \\
\hline & Radiotherapy & $18(7.2)$ & $9(8.3)$ & $2(7.4)$ & $6(7.7)$ & $1(2.6)$ & \\
\hline & Chemotherapy & $49(19.5)$ & $20(18.5)$ & $2(7.4)$ & $18(23.1)$ & $9(23.7)$ & \\
\hline & Chemoradiotherapy & $6(2.4)$ & $2(1.9)$ & $1(1.3)$ & $1(1.3)$ & $2(5.3)$ & \\
\hline \multirow[t]{5}{*}{ ASA class } & & & & & & & 0.70 \\
\hline & 1 & $70(27.9)$ & $26(24.1)$ & $11(40.7)$ & $22(28.2)$ & $11(28.9)$ & \\
\hline & 2 & $163(64.9)$ & $73(67.6)$ & $13(48.1)$ & $52(66.7)$ & $25(65.8)$ & \\
\hline & 3 & $18(7.2)$ & $9(8.3)$ & $3(11.1)$ & $4(5.1)$ & $2(5.3)$ & \\
\hline & $4-5$ & $0(0)$ & $0(0)$ & $0(0)$ & $0(0)$ & $0(0)$ & \\
\hline
\end{tabular}

Result(s)* Molecular analysis was successful in 367 EC; 251 patients had undergone LND, see table 1. Median follow-up was 11 years (range 7.5-15.4). Multivariable analysis showed that molecular subgroup was a strong independent prognostic factor for recurrence: p53abn HR 3.88 (95\%CI 1.89-7.94, $p<0.001)$ and NSMP HR 4.80 (95\%CI 2.14-10.78, p<0.001) compared to MMRd. Figure $1 \mathrm{~A}$ shows time to recurrence among those patients staged by LND as IA-IB by molecular subgroup.

Among 264 patients without AT, 247 (94\%) had stage I-II disease and $17(6 \%)$ stage III. None of the patients with POLEmut EC $(\mathrm{n}=26,10 \%)$ had a recurrence (figure $1 \mathrm{~B})$. Multivariable analysis showed that the significant prognostic impact of molecular subgroup was independent of clinicopathological factors.

Conclusion* The molecular EC classification has strong prognostic value, independent of clinicopathological factors, also among patients staged by LND and those without AT. This implies that the unfavourable prognosis of p53abn EC is not caused by undetected lymph node metastasis. POLEmut EC is inherently associated with an excellent prognosis even in the absence of adjuvant treatment.

\section{PERFORMANCE OF DNA METHYLATION ANALYSIS IN URINE, CERVICOVAGINAL SELF-SAMPLES AND CERVICAL SCRAPES FOR ENDOMETRIAL CANCER DETECTION}

${ }^{1 ; 2} \mathrm{R}$ Van den Helder*, ${ }^{2} \mathrm{BMM}$ Wever, ${ }^{2} \mathrm{AP}$ Van Splunter, ${ }^{3} \mathrm{CH}$ Mom, ${ }^{3} \mathrm{~J}$ Kasius, ${ }^{2}$ RDM Steenbergen, ${ }^{1} \mathrm{~N}$ Van Trommel, ${ }^{2} \mathrm{MCG}$ Bleeker. ${ }^{7}$ The Netherlands Cancer Institute (NKI), Department of Gynecologic Oncology, Amsterdam, Netherlands; ${ }^{2}$ Amsterdam University Medical Center (UMC), Vrije Universiteit Amsterdam, Department of Pathology, Amsterdam, Netherlands; ${ }^{3}$ Amsterdam UMC, locatie AMC, Department of Gynecologic Oncology, Amsterdam, Netherlands

10.1136/ijgc-2021-ESGO.139 